Int. J. Open Problems Compt. Math., Vol. 6, No. 4, December 2013

ISSN 1998-6262; Copyright (CICSRS Publication, 2013

www.i-csrs.org

\title{
Convergent Iterative Algorithms in the 2-inner Product Space $\mathbb{R}^{n}$
}

\author{
Iqbal H. Jebril and Mustapha Raïssouli \\ Taibah-University, Faculty of Science, Department of Mathematics, \\ Al Madinah Al Munawwarah, P.O. Box 30097, Zip Code 41477, \\ Kingdom of Saudi Arabia. \\ e-mail: iqbal501@hotmail.com and raissouli_10@hotmail.com
}

\begin{abstract}
In this paper, some iterative algorithms in the 2-inner product space $\mathbb{R}^{n}$ are constructed and studied. Open problems are derived as well.
\end{abstract}

Keywords: 2-Norm, 2-Inner Product, Duality, Iterative Algorithms, PointWise Convergence

2010 Mathematics Subject Classification: 41A15, 41A65.

\section{Introduction}

The concepts of linear 2-normed spaces and 2-inner product spaces have been extensively studied and developed in different subjects by many authors, see $[1,2,3]$ for instance and the related references cited therein.

In this section, we will recall some basic notions and results needed throughout the paper. Let $X$ be a linear space of dimension greater than 1 over the field $\mathbb{K}=\mathbb{R}$ or $\mathbb{K}=\mathbb{C}$. Let $\|.,$.$\| be a real-valued function on X \times X$ satisfying the following conditions:

$\left(2 n_{1}\right)\|x, y\|=0$ if and only if $x$ and $y$ are linearly dependent, $\left(2 n_{2}\right)\|x, y\|=\|y, x\|$ for all $x, y \in X$,

$\left(2 n_{3}\right)\|\lambda x, y\|=|\lambda|\|x, y\|$ for all $\lambda \in \mathbb{K}$ and all $x, y \in X$,

$\left(2 n_{4}\right)\|x+y, z\| \leq\|x, z\|+\|y, z\|$ for all $x, y, z \in X$. 
Then $\|.,$.$\| is called a 2$-norm on $X$ and $(X,\|.,\|$.$) is called a 2-normed space.$ Some of the basic properties of the 2-norms are that they are non-negative and $\|x, y+\alpha x\|=\|x, y\|$ for every $x, y \in X$ and $\alpha \in \mathbb{K}$.

Under the same assumptions over $X$, let $(., . \mid$.) be a $\mathbb{K}$-valued function defined on $X \times X \times X$ satisfying the following conditions: $\left(2 i_{1}\right)(x, x \mid z) \geq 0$ for all $x, z \in X$, and, $(x, x \mid z)=0$ if and only if $x$ and $z$ are linearly dependent, $\left(2 i_{2}\right)(x, x \mid z)=(z, z \mid x)$ for all $x, z \in X$, $\left(2 i_{3}\right)(x, y \mid z)=(y, x \mid z)$ for all $x, y, z \in X$, $\left(2 i_{4}\right)(\lambda x, y \mid z)=\lambda(x, y \mid z)$ for all $\lambda \in \mathbb{K}$ and all $x, y, z \in X$, $\left(2 i_{5}\right)\left(x_{1}+x_{2}, y \mid z\right)=\left(x_{1}, y \mid z\right)+\left(x_{2}, y \mid z\right)$ for all $x_{1}, x_{2}, y, z \in X$.

Then $(., . \mid$.$) is called a 2$-inner product on $X$ and $(X,(., . \mid)$.$) is called a 2-inner$ product space. Whenever a 2 -inner product $(., . \mid$.$) on X$ is given, we can define an associate 2-norm on $X$ defined by

$$
\forall x, z \in X \quad\|x, z\|=\sqrt{(x, x \mid z)}
$$

Some basic properties of the 2 -inner product (.,.|.) are recited in the following.

(i) For all $x, y \in X$, one has $(x, y \mid y)=0$ and $(x, y \mid 0)=0$

(ii) The analogue of the standard Cauchy-Schwartz inequality holds, i.e. for all $x, y, z \in X$,

$$
|(x, y \mid z)| \leq \sqrt{(x, x \mid z)} \sqrt{(y, y \mid z)}=\|x, z\|\|y, z\|
$$

(iii) If $(X,(. \mid$.$) is an inner product space, then we can define a 2$-inner product $(., . \mid$.$) on X$ by setting

$$
\forall x, y, z \in X \quad(x, y \mid z)=(x \mid y)\|z\|^{2}-(x \mid z)(y \mid z)
$$

Let $\mathbb{R}^{n}$ be the classical finite dimensional space with $n \geq 2$. By analogy with its familiar norms, $\mathbb{R}^{n}$ can be equipped with the following standard 2norms: For all $x=\left(x_{1}, x_{2}, \ldots, x_{n}\right) \in \mathbb{R}^{n}$ and $y=\left(y_{1}, y_{2}, \ldots, y_{n}\right) \in \mathbb{R}^{n}$, we get

$$
\begin{gathered}
\|x, y\|_{1}=\sum_{1 \leq i<j \leq n}\left|x_{i} y_{j}-x_{j} y_{i}\right|, \\
\|x, y\|_{2}=\left(\sum_{1 \leq i<j \leq n}\left(x_{i} y_{j}-x_{j} y_{i}\right)^{2}\right)^{1 / 2}, \\
\|x, y\|_{\infty}=\max _{1 \leq i<j \leq n}\left|x_{i} y_{j}-x_{j} y_{i}\right| .
\end{gathered}
$$

The above 2-norm $\|., .\|_{2}$ derives from the classical 2-inner product of $\mathbb{R}^{n}$, socalled the euclidian 2-norm (resp. 2-inner product) of $\mathbb{R}^{n}$, defined as follows

$$
(x, y \mid z)=\sum_{1 \leq i<j \leq n}\left(x_{i} z_{j}-x_{j} z_{i}\right)\left(y_{i} z_{j}-y_{j} z_{i}\right)
$$


for all $x=\left(x_{1}, x_{2}, \ldots, x_{n}\right) \in \mathbb{R}^{n}, y=\left(y_{1}, y_{2}, \ldots, y_{n}\right) \in \mathbb{R}^{n}$ and $z=\left(z_{1}, z_{2}, \ldots, z_{n}\right) \in$ $\mathbb{R}^{n}$.

Generally, if $p \in[1, \infty]$ is an extended real number and we set

$$
\|x, y\|_{p}=\left(\sum_{1 \leq i<j \leq n}\left|x_{i} y_{j}-x_{j} y_{i}\right|^{p}\right)^{1 / p},
$$

then $\|., .\|_{p}$ is a 2 -norm on $\mathbb{R}^{n}$, called power 2 -norm of $\mathbb{R}^{n}$. This power 2 -norm includes some of the most standard 2-norms of $\mathbb{R}^{n}$. Namely, $\|., .\|_{p}$ gives $\|., .\|_{1}$ and $\|., .\|_{2}$ when we take $p=1$ and $p=2$ respectively, and $\|., .\|_{p}$ extends also $\|., .\|_{\infty}$ in the following sense

$$
\forall x, y \in \mathbb{R}^{n} \quad\|x, y\|_{\infty}=\lim _{p \rightarrow \infty}\|x, y\|_{p}
$$

For $p \in[1, \infty]$, we denote by $p^{*}$ its conjugate defined by $1 / p+1 / p^{*}=1$ i.e. $p^{*}=p / p-1$ with the convention, if $p=1$ then $p^{*}=\infty$ and if $p=\infty$ then $p^{*}=1$. With this, the above power 2-norm $\|., .\|_{p}$ of $\mathbb{R}^{n}$ satisfies the following properties:

- An analogue of Hölder inequality holds, i.e. for all $x, y, z \in \mathbb{R}^{n}$ one has

$$
|(x, y \mid z)| \leq\|x, z\|_{p}\|y, z\|_{p^{*}} .
$$

For $p=2$, this inequality remains the Cauchy-Schwartz one.

- For all $x, y \in \mathbb{R}^{n}$ we have

$$
\|x, y\|_{p} \leq \frac{1}{p}\|x, y\|_{1}+\frac{1}{p^{*}}\|x, y\|_{\infty} .
$$

In particular, for all $x, y \in \mathbb{R}^{n}$ one has

$$
2\|x, y\|_{2} \leq\|x, y\|_{1}+\|x, y\|_{\infty} .
$$

- The map $p \longmapsto\|.,\|_{p}$ is point-wisely monotone decreasing on $[1, \infty[,[4]$, in the sense that, for all $x, y \in \mathbb{R}^{n}$ there holds

$$
p \geq q \geq 1 \Longrightarrow\|x, y\|_{p} \leq\|x, y\|_{q} .
$$

In particular, the following double inequality

$$
\|x, y\|_{\infty} \leq\|x, y\|_{2} \leq\|x, y\|_{1},
$$

holds for all $x, y \in \mathbb{R}^{n}$.

The remainder of this paper will be organized as follows: After this introduction, Section 2 turns out of the duality notion of maps together with some properties that will be needed in the sequel. Section 3 is focused to construct an iterative algorithm converging, for the point-wise topology, to the euclidian 2-norm of $\mathbb{R}^{n}$. In Section 4, we present a generalized algorithm, extending the above one and we study its point-wise convergence. This section will be ended by summarizing the above results in the aim to put some open problems. 


\section{Duality in the 2-Inner Product $\mathbb{R}^{n}$}

We preserve the same notations as in the previous section. We precise here some additional notions. Let $b \in \mathbb{R}^{n}$ with $b \neq 0$. The notation $<b>$ refers to the subspace of $\mathbb{R}^{n}$ generated by $b$ i.e. $\langle b>=\{t b, t \in \mathbb{R}\}$. With this, we may state the following.

Definition 2.1 Let $\theta(.,):. \mathbb{R}^{n} \times \mathbb{R}^{n} \longrightarrow[0,+\infty]$ be a map satisfying the condition $\left(2 n_{1}\right)$ and let $b \in \mathbb{R}^{n}$ with $b \neq 0$.

- The b-dual of $\theta(.,$.$) is the real-valued map \theta_{b}^{*}: \mathbb{R}^{n} \longrightarrow[0,+\infty]$ defined by

$$
\forall x \in \mathbb{R}^{n} \quad \theta_{b}^{*}(x)=\sup _{y \notin<b>} \frac{|(x, y \mid b)|}{\theta(y, b)} .
$$

- The pseudo-dual of $\theta(.,$.$) is the map \theta^{*}(.,):. \mathbb{R}^{n} \times \mathbb{R}^{n} \longrightarrow[0,+\infty]$ defined by

$$
\forall x, z \in \mathbb{R}^{n} \quad \theta^{*}(x, z)=\theta_{z}^{*}(x) \text { if } z \neq 0 \text { and } \theta^{*}(x, 0)=0 .
$$

We explicitly notice that the above maps $\theta(.,),. \theta_{b}^{*}($.$) and \theta^{*}(.,$.$) can take the$ value $+\infty$, with the convention $1 /+\infty=0$ and $0 / 0=+\infty$. It is easy to see that $\theta_{b}^{*}(x)=0$ if and only if $x \in<b>$, that is, $\theta^{*}(.,$.$) satisfies the condition$ $\left(2 n_{1}\right)$. If $\theta(.,$.$) is a 2$-norm of $\mathbb{R}^{n}$ with pseudo-dual $\theta^{*}(.,$.$) also a 2$-norm, we say that $\theta(.,$.$) is a regular 2$-norm of $\mathbb{R}^{n}$. As we shall see later, all the above standard 2-norms of $\mathbb{R}^{n}$ are regular.

The elementary properties of the duality notion are summarized in the following.

Proposition 2.1 With the above, the following assertions are met.

1. For all $x \in \mathbb{R}^{n}$,

$$
\theta_{b}^{*}(x)=\sup \left\{\frac{|(x, y \mid b)|}{\theta(y, b)}, \theta(y, b)>0\right\}=\sup \left\{\frac{|(x, y \mid b)|}{\theta(y, b)}, 0<\theta(y, b)<+\infty\right\} .
$$

2. For all $x, z \in \mathbb{R}^{n}$,

$$
\theta^{*}(x, z)=\sup \left\{|(x, y \mid z)| ; \quad y \in \mathbb{R}^{n}, \theta(y, z) \leq 1\right\} .
$$

3. For all $x, y, z \in \mathbb{R}^{n}$,

$$
|(x, y \mid z)| \leq \theta^{*}(x, z) \theta(y, z) .
$$

In particular, for all $x, z \in \mathbb{R}^{n}$ we have

$$
\|x, z\|_{2}^{2} \leq \theta(x, z) \theta^{*}(x, z) .
$$

4. For all $\theta(.,$.$) satisfying \left(2 n_{1}\right)$ one has

$$
\theta^{* *}(., .):=\left(\theta^{*}\right)^{*}(., .) \leq \theta(., .) .
$$


Proof. It is straightforward and we omit the details here.

The set of all (extended) real maps defined on $\mathbb{R}^{n} \times \mathbb{R}^{n}$ into $[0,+\infty]$ can be equipped with a partial ordering, called point-wise order, defined by the following way. For $\theta_{1}(.,$.$) and \theta_{2}(.,$.$) , we write \theta_{1}(.,.) \leq \theta_{2}(.,$.$) if and only if$ $\theta_{1}(x, y) \leq \theta_{2}(x, y)$ for all $x, y \in \mathbb{R}^{n}$, where we extend the classical order of $\mathbb{R}$ to $\mathbb{R} \cup\{+\infty\}$ by setting $t \leq+\infty$ for all $t \in \mathbb{R} \cup\{+\infty\}$. With this it is easy to see that, if $\theta_{1}(.,$.$) and \theta_{2}(.,$.$) are two maps satisfying the condition \left(2 n_{1}\right)$ with $\theta_{1}(.,.) \leq \theta_{2}(.,$.$) then \theta_{1}^{*}(.,.) \geq \theta_{2}^{*}(.,$.$) .$

Proposition 2.2 The Euclidian 2-norm $\|., .\|_{2}$ of $\mathbb{R}^{n}$ is regular and self-dual, that is,

$$
\forall x, z \in \mathbb{R}^{n} \quad\|x, z\|_{2}^{*}=\|x, z\|_{2} .
$$

Further, $\|.,\|_{2}$ is the unique self-dual 2-norm of $\mathbb{R}^{n}$.

Proof. If $x$ and $z$ are linearly dependent then $\|x, z\|_{2}^{*}=\|x, z\|_{2}=0$. Assume that below $x$ and $z$ are linearly independent. By definition with CauchySchwartz inequality we obtain

$$
\|x, z\|_{2}^{*}:=\sup \left\{\frac{|(x, y \mid z)|}{\|y, z\|_{2}} ; y \in \mathbb{R}^{n},\|y, z\|_{2}>0\right\} \leq\|x, z\|_{2} .
$$

Moreover, in (2.2) the upper bound $\|x, z\|_{2}$ is attained by the "sup" for $y=$ $\frac{x}{\|x, z\|_{2}}$. It follows that $\|.,\|_{2}$ is a regular and self-dual 2-norm of $\mathbb{R}^{n}$.

Now, let $\theta(.,$.$) be a self-dual 2-norm of \mathbb{R}^{n}$, we will prove that $\theta(.,)=.\|., .\|_{2}$. Indeed, according to inequality $(2.1)$, with the fact that $\theta^{*}(x, z)=\theta(x, z)$ for all $x, z \in \mathbb{R}^{n}$, we deduce that

$$
\forall x, z \in \mathbb{R}^{n} \quad \theta(x, z) \geq\|x, z\|_{2},
$$

which, by taking the pseudo-dual, implies that

$$
\forall x, z \in \mathbb{R}^{n} \quad \theta^{*}(x, z)=\theta(x, z) \leq\|x, z\|_{2}^{*}=\|x, z\|_{2} .
$$

The desired result follows so completes the proof.

Proposition 2.3 For all $p \in[1, \infty]$, the power 2 -norm $\|.,\|_{p}$ of $\mathbb{R}^{n}$ is regular with the relationship

$$
\|., .\|_{p}^{*}=\|., .\|_{p^{*}} .
$$

In particular, $\|., .\|_{1}$ and $\|., .\|_{\infty}$ are regular and mutually dual. Again $\left\|_{., .}\right\|_{2}$ is regular and self-dual. 
Proof. Analogously to the proof of Proposition 2.2 we can assume that $x$ and $z$ are linearly independent. It is well known that the Hölder inequality is the best possible, that is,

$$
\sup \left\{\frac{|(x, y \mid z)|}{\|y, z\|_{p}} ; \quad y \in \mathbb{R}^{n},\|y, z\|_{p}>0\right\}=\|x, z\|_{p^{*}} .
$$

We then deduce that $\|., .\|_{p}^{*}=\|., .\|_{p^{*}}$ and so $\|., .\|_{p}^{*}$ is a 2-norm then $\|., .\|_{p}$ is regular. Taking $p=1, p=+\infty$ and $p=2$ we obtain the rest of the proposition. The proof is so complete.

Proposition 2.4 Let $\theta_{1}(.,):. \mathbb{R}^{n} \times \mathbb{R}^{n} \longrightarrow[0,+\infty]$ and $\theta_{2}(.,):. \mathbb{R}^{n} \times \mathbb{R}^{n} \longrightarrow$ $[0,+\infty]$ be two maps satisfying the condition $\left(2 n_{1}\right)$ and let $\left.p \in\right] 1, \infty[$. Then there holds

$$
\left(\frac{1}{p} \theta_{1}(., .)+\frac{1}{p^{*}} \theta_{2}(., .)\right)^{*} \leq \frac{1}{p} \theta_{1}^{*}(., .)+\frac{1}{p^{*}} \theta_{2}^{*}(., .) .
$$

Proof. By definition, for all $x, z \in \mathbb{R}^{n}$ with $z \neq 0$ we have

$$
\begin{aligned}
& \left(\frac{1}{p} \theta_{1}(., .)+\frac{1}{p^{*}} \theta_{2}(., .)\right)^{*}(x, z) \\
= & \sup \left\{\frac{|(x, y \mid z)|}{\frac{1}{p} \theta_{1}(y, z)+\frac{1}{p^{*}} \theta_{2}(y, z)} ; y \in \mathbb{R}^{n}, 0<\theta_{1}(y, z)<+\infty, 0<\theta_{2}(y, z)<+\infty\right\} .
\end{aligned}
$$

By convexity of the real-mapping $t \longmapsto 1 / t, t \in] 0,+\infty[$, one has

$$
\frac{|(x, y \mid z)|}{\frac{1}{p} \theta_{1}(y, z)+\frac{1}{p^{*}} \theta_{2}(y, z)} \leq \frac{1}{p} \frac{|(x, y \mid z)|}{\theta_{1}(y, z)}+\frac{1}{p^{*}} \frac{|(x, y \mid z)|}{\theta_{2}(y, z)} .
$$

Combining (2.5) and (2.6), the desired result follows by the subadditivity of the "sup" with an elementary manipulation, thus completes the proof.

We end this section by introducing some notions needed later. Let $\left(\theta^{k}(., .)\right)_{k}$ be a sequence of real maps defined on $\mathbb{R}^{n} \times \mathbb{R}^{n}$. We say that $\left(\theta^{k}(., .)\right)_{k}$ converges point-wisely to a map $\theta(.,$.$) if for all x, y \in \mathbb{R}^{n}, \theta^{k}(x, y)$ converges (in $\mathbb{R}$ ) to $\theta(x, y)$. The map $\theta(.,$.$) will be called the point-wise limit of the sequence$ $\left(\theta^{k}(., .)\right)_{k}$.

We say that $\left(\theta^{k}(., .)\right)_{k}$ is point-wisely monotone decreasing if and only if

$$
\theta^{k+1}(x, y) \leq \theta^{k}(x, y)
$$

for all $k \geq 0$ and all $x, y \in \mathbb{R}^{n}$. The following result, needed in the sequel, may be stated. 
Proposition 2.5 Let $\left(\theta^{k}(., .)\right)_{k}$ be a sequence of maps defined from $\mathbb{R}^{n} \times \mathbb{R}^{n}$ into $[0,+\infty]$ such that:

(i) $\left(\theta^{k}(., .)\right)_{k}$ converges point-wisely to a map $\theta(.,$.$) satisfying the condition$ $\left(2 n_{1}\right)$,

(ii) For all $k \geq 0$, the map $\theta^{k}(.,$.$) satisfies the condition \left(2 n_{1}\right)$,

(iii) $\left(\theta^{k}(., .)\right)_{k}$ is point-wise monotone decreasing.

Then $\left(\left(\theta^{k}\right)^{*}(., .)\right)_{k}$ is increasingly convergent point-wisely to $\theta^{*}(.,$.$) .$

Proof. Since the sequence $\left(\theta^{k}(., .)\right)_{k}$ satisfies (ii) and (iii) then, for all $x, z \in \mathbb{R}^{n}$ with $z \neq 0$, we have successively

$$
\theta^{*}(x, z)=\sup _{\theta(y, z)>0} \frac{|(x, y \mid z)|}{\theta(y, z)}=\sup _{\theta(y, z)>0} \frac{|(x, y \mid z)|}{\inf _{k \geq 0} \theta^{k}(y, z)}
$$

or again

$$
\theta^{*}(x, z)=\sup _{\theta(y, z)>0} \sup _{k \geq 0} \frac{|(x, y \mid z)|}{\theta^{k}(y, z)}=\sup _{k \geq 0} \sup _{\theta(y, z)>0} \frac{|(x, y \mid z)|}{\theta^{k}(y, z)} .
$$

It is easy to verify that $\theta(y, z)>0$ if and only if $\theta^{k}(y, z)>0$ for each $k \geq 0$. So we deduce that

$$
\theta^{*}(x, z)=\sup _{k \geq 0} \sup _{\theta^{k}(y, z)>0} \frac{|(x, y \mid z)|}{\theta^{k}(y, z)}=\sup _{k \geq 0}\left(\theta^{k}\right)^{*}(x, z) .
$$

The fact that $\left(\theta^{k}(.,).\right)$ is point-wisely decreasing implies that $\left(\left(\theta^{k}\right)^{*}(., .)\right)_{k}$ is point-wise incresing, the desired result follows and the proof is complete.

Remark 2.1 We left the reader to verify throughout a counter-example that the conclusion of the above proposition does not hold if the sequence $\left(\theta^{k}(., .)\right)_{k}$ is point-wise monotone increasing.

\section{Algorithm Converging to the 2-Norm $\|., .\|_{2}$ of $\mathbb{R}^{n}$}

For all $x, y \in \mathbb{R}^{n}$, let us define the following recursive process

$$
\Lambda^{0}(x, y)=\|x, y\|_{1}, \quad \Lambda^{k+1}(x, y)=\frac{1}{2} \Lambda^{k}(x, y)+\frac{1}{2}\left(\Lambda^{k}\right)^{*}(x, y), k \geq 0 .
$$

Theorem 3.1 With the above, the sequence $\left(\Lambda^{k}\right)_{k}$ converges point-wisely to the euclidian 2-norm of $\mathbb{R}^{n}$, i.e.

$$
\forall x, y \in \mathbb{R}^{n} \quad\|x, y\|_{2}=\lim _{k \rightarrow \infty} \Lambda^{k}(x, y) .
$$


Moreover, the following estimation inequality holds

$$
\forall k \geq 0, \forall x, y \in \mathbb{R}^{n} \quad 0 \leq \Lambda^{k}(x, y)-\|x, y\|_{2} \leq \frac{1}{2^{k}}\left(\|x, y\|_{1}-\|x, y\|_{\infty}\right) .
$$

Proof. If $x$ and $y$ are linearly dependent then the desired result is immediate. Assume that $x$ and $y$ are linearly independent. By (2.1) with (3.1), we have

$$
\Lambda^{k+1}(x, y) \geq \frac{1}{2} \Lambda^{k}(x, y)+\frac{1}{2} \frac{\mid(x, x|y|}{\Lambda^{k}(x, y)} .
$$

By the increase monotonicity and concavity of the real-map $t \longmapsto \ln t, t \in$ ] $0,+\infty[$, inequality (3.2) implies that

$$
\ln \Lambda^{k+1}(x, y) \geq \frac{1}{2} \ln \Lambda^{k}(x, y)+\frac{1}{2}\left(\ln |(x, x \mid y)|-\ln \Lambda^{k}(x, y)\right),
$$

or again, after a simple reduction

$$
\ln \Lambda^{k+1}(x, y) \geq \frac{1}{2} \ln |(x, x \mid y)|=\ln \|x, y\|_{2} .
$$

This, with the fact that $\|x, y\|_{2} \leq\|x, y\|_{1}:=\Lambda^{0}(x, y)$, yields

$$
\Lambda^{k}(x, y) \geq\|x, y\|_{2}
$$

for all $k \geq 0$. Since $\|., .\|_{2}$ is self-dual, the above inequality implies the following one

$$
\left(\Lambda^{k}\right)^{*}(x, y) \leq\|x, y\|_{2}
$$

for all $k \geq 0$. Now, we have

$$
\begin{aligned}
0 \leq \Lambda^{k+1}(x, y)-\|x, y\|_{2}=\frac{1}{2}\left(\Lambda^{k}(x, y)-\|x, y\|_{2}\right) & +\frac{1}{2}\left(\left(\Lambda^{k}\right)^{*}(x, y)-\|x, y\|_{2}\right) \\
& \leq \frac{1}{2}\left(\Lambda^{k}(x, y)-\|x, y\|_{2}\right)
\end{aligned}
$$

from which, by mathematical induction on $k \geq 0$, we deduce that

$$
0 \leq \Lambda^{k}(x, y)-\|x, y\|_{2} \leq \frac{1}{2^{k}}\left(\|x, y\|_{1}-\|x, y\|_{2}\right),
$$

for all $k \geq 0$ and all $x, y \in \mathbb{R}^{n}$. The desired result follows from the fact that $\|x, y\|_{\infty} \leq\|x, y\|_{2}$, so completes the proof.

Remark 3.1 Using Proposition 2.4 with Proposition 2.1,3., another different proof for showing the above theorem is possible. For analogue idea, see the proof of Theorem 4.2 below. We left the details here. 
Remark 3.2 With the above, let us set, for all $x, y \in \mathbb{R}^{n}$

$$
E^{k}(x, y)=\Lambda^{k}(x, y)-\|x, y\|_{2},
$$

called the error estimation for the point-wise convergence of $\left(\Lambda^{k}\right)_{k}$ to $\|., .\|_{2}$. Following the above proof, it is proved that

$$
0 \leq E^{k+1}(x, y) \leq \frac{1}{2} E^{k}(x, y) \leq \ldots \leq \frac{1}{2^{k}} E^{0}(x, y) .
$$

That is to say that $\left(\Lambda^{k}\right)_{k}$ converges point-wisely to $\|.,\|_{2}$ with a geometric speed.

\section{Generalized Iterative Algorithm}

As already pointed out, this section investigates a converging algorithm extending the above one. Let $p \in[1, \infty]$ be as in the above and define the sequence $\left(\Upsilon_{p}^{k}(., .)\right)_{k}$ by the following iterate process

$$
\Upsilon_{p}^{k+1}(x, y)=\frac{1}{p} \Upsilon_{p}^{k}(x, y)+\frac{1}{p^{*}}\left(\Upsilon_{p^{*}}^{k}\right)^{*}(x, y), k \geq 0 ; \quad \Upsilon_{p}^{0}(x, y)=\|x, y\|_{1}
$$

It is easy to see that, for all $p \in[1, \infty]$ and $k \geq 0$, the map $\Upsilon_{p}^{k}(.,$.$) is defined$ from $\mathbb{R}^{n} \times \mathbb{R}^{n}$ into $\left[0,+\infty\left[\right.\right.$. Further $\Upsilon_{p}^{k}(.,$.$) satisfies the condition \left(2 n_{1}\right)$ and so the above algorithm is recursively well defined. Let us remark that

$$
\Upsilon_{p}^{1}(x, y)=\frac{1}{p}\|x, y\|_{1}+\frac{1}{p^{*}}\|x, y\|_{\infty}
$$

and, $\Upsilon_{1}^{k}(x, y)=\|x, y\|_{1}, \Upsilon_{\infty}^{k}(x, y)=\|x, y\|_{\infty}$ for all $k \geq 0$. Below, we can then assume that $1<p<\infty$. Clearly, for $p=2$, algorithm (4.1) coincides with (3.1).

Proposition 4.1 For all $1<p<\infty$, the following assertions are met:

1. For every $k \geq 0$ and all $x, y \in \mathbb{R}^{n},\left(\Upsilon_{p^{*}}^{k}\right)^{*}(x, y) \leq \Upsilon_{p}^{k}(x, y)$.

2. The sequence $\left(\Upsilon_{p}^{k}(., .)\right)_{k}$ is point-wisely monotonic decreasing.

\section{Proof.}

1. For $k=0$ it is trivial. By Proposition 2.4 with Proposition 2.1,3., we obtain for all $x, y \in \mathbb{R}^{n}$,

$$
\left(\Upsilon_{p^{*}}^{k+1}\right)^{*}(x, y) \leq \frac{1}{p^{*}}\left(\Upsilon_{p^{*}}^{k}\right)^{*}(x, y)+\frac{1}{p} \Upsilon_{p}^{k}(x, y)=\Upsilon_{p}^{k+1}(x, y),
$$

which gives the desired inequality.

2. Substituting the above inequality in (4.1), we immediately deduce the point-wise decreasing monotonicity of the sequence $\left(\Upsilon_{p}^{k}(., .)\right)_{k}$. The proof is thus complete. 
Theorem 4.2 The sequence $\left(\Upsilon_{p}^{k}(., .)\right)_{k}$ converges point-wisely to a map $\Upsilon_{p}(.,$. satisfying the condition $\left(2 n_{1}\right)$. Furthermore, the following estimation

$$
0 \leq \Upsilon_{p}^{k}(x, y)-\Upsilon_{p}(x, y) \leq \frac{1}{p^{k}}\left(\|x, y\|_{1}-\|x, y\|_{\infty}\right)
$$

holds for all $k \geq 0$ and all $x, y \in \mathbb{R}^{n}$.

Proof. According to Proposition 4.1, we have

$$
\begin{aligned}
\|x, y\|_{\infty} \leq \ldots \leq\left(\Upsilon_{p^{*}}^{k-1}\right)^{*}(x, y) & \leq\left(\Upsilon_{p^{*}}^{k}\right)^{*}(x, y) \\
& \leq \Upsilon_{p}^{k}(x, y) \leq \Upsilon_{p}^{k-1}(x, y) \leq \ldots \leq\|x, y\|_{1}
\end{aligned}
$$

for all $k \geq 0$ and all $x, y \in \mathbb{R}^{n}$. It follows that, for all $x, y \in \mathbb{R}^{n}$, the real sequences $\left(\Upsilon_{p}^{k}(x, y)\right)_{k}$ is monotone decreasing and lower bounded, so it is convergent in $\mathbb{R}$. Proposition 2.5, with $(4.4)$, tells us that $\left(\Upsilon_{p}^{k}(., .)\right)_{k}$ converges point-wisely to a map denoted by $\Upsilon_{p}(.,$.$) and satisfying condition \left(2 n_{1}\right)$. To prove the estimation (4.3), we first remark that,

$$
\left(\Upsilon_{p^{*}}^{k}\right)^{*}(x, y) \leq \Upsilon_{p}(x, y) \leq \Upsilon_{p}^{k}(x, y)
$$

which with the fact that

$$
\Upsilon_{p}^{k+1}(x, y)-\Upsilon_{p}(x, y)=\frac{1}{p}\left(\Upsilon_{p}^{k}(x, y)-\Upsilon_{p}(x, y)\right)+\frac{1}{p^{*}}\left(\left(\Upsilon_{p^{*}}^{k}\right)^{*}(x, y)-\Upsilon_{p}(x, y)\right)
$$

yields the following double inequality

$$
0 \leq \Upsilon_{p}^{k+1}(x, y)-\Upsilon_{p}(x, y) \leq \frac{1}{p}\left(\Upsilon_{p}^{k}(x, y)-\Upsilon_{p}(x, y)\right),
$$

for all $k \geq 0$ and all $x, y \in \mathbb{R}^{n}$. The desired result follows by a mathematical induction with a simple manipulation. The proof of the theorem is complete.

Corollary 4.3 For every $1<p<\infty$ and all $x, y \in \mathbb{R}^{n}$, the following properties hold

(i) $\Upsilon_{p}(x, y) \leq \frac{1}{p}\|x, y\|_{1}+\frac{1}{p^{*}}\|x, y\|_{\infty}$.

(ii) $\left(\Upsilon_{p}\right)^{*}(x, y)=\Upsilon_{p^{*}}(x, y)$. In particular, one has $\Upsilon_{2}(x, y)=\|x, y\|_{2}$.

\section{Proof.}

(i) By the point-wise decrease monotonicity of $\left(\Upsilon_{p}^{k}(., .)\right)_{k}$ we have

$$
\Upsilon_{p}^{k}(x, y) \leq \Upsilon_{p}^{1}(x, y)=\frac{1}{p}\|x, y\|_{1}+\frac{1}{p^{*}}\|x, y\|_{\infty} .
$$


Letting $k \rightarrow \infty$ in this latter inequality we obtain the desired result.

(ii) Since $\left(\Upsilon_{p}^{k}(., .)\right)_{k}$ converges point-wisely to $\Upsilon_{p}(.,$.$) for every 1<p<\infty$ then $\left(\Upsilon_{p^{*}}^{k}(., .)\right)_{k}$ converges point-wisely to $\Upsilon_{p^{*}}(.,$.$) . Algorithm (4.1), with Proposi-$ tion 2.5, yields when $k \rightarrow \infty$ the first relation of (ii). In particular, if $p=2$ then $p^{*}=2$ and so $\left(\Upsilon_{2}(., .)\right)^{*}=\Upsilon_{2}(.,$.$) . Since \|., .\|_{2}$ is the unique self-dual 2 -norm of $\mathbb{R}^{n}$, the second relation of (ii) is so obtained, thus concludes the proof.

Proposition 4.4 For $1<p<\infty$, the map $p \longmapsto \Upsilon_{p}(.,$.$) is point-wisely$ monotone decreasing. That is, for all $x, y \in \mathbb{R}^{n}$ we have

$$
p \geq q>1 \Longrightarrow \Upsilon_{p}(x, y) \leq \Upsilon_{q}(x, y) .
$$

Proof. By a mathematical induction on $k \geq 1$, we wish to establish that, for all $x, y \in \mathbb{R}^{n}$, we have $\Upsilon_{p}^{k}(x, y) \leq \Upsilon_{q}^{k}(x, y)$ whenever $p \geq q$. For $k=1$, it is immediate from the decrease monotonicity of $p \longmapsto \frac{1}{p}\|x, y\|_{1}+\frac{1}{p^{*}}\|x, y\|_{\infty}$, since $\|x, y\|_{\infty} \leq\|x, y\|_{1}$. Now, assume that for $p$ and $q$ such that $p \geq q$ we have $\Upsilon_{p}^{k}(x, y) \leq \Upsilon_{q}^{k}(x, y)$ for all $x, y \in \mathbb{R}^{n}$. One has

$$
\Upsilon_{p}^{k+1}(x, y)=\frac{1}{p} \Upsilon_{p}^{k}(x, y)+\frac{1}{p^{*}}\left(\Upsilon_{p^{*}}^{k}\right)^{*}(x, y) \leq \frac{1}{p} \Upsilon_{q}^{k}(x, y)+\frac{1}{p^{*}}\left(\Upsilon_{q^{*}}^{k}\right)^{*}(x, y)
$$

According to Proposition 4.1, we have $\left(\Upsilon_{q^{*}}^{k}\right)^{*}(x, y) \leq \Upsilon_{q}^{k}(x, y)$ which, with Proposition 2.4 and Proposition 2.1,3., yields

$$
\Upsilon_{p}^{k+1}(x, y) \leq \frac{1}{q} \Upsilon_{q}^{k}(x, y)+\frac{1}{q^{*}}\left(\Upsilon_{q^{*}}^{k}\right)^{*}(x, y)=\Upsilon_{q}^{k+1}(x, y)
$$

Summarizing, for $k \geq 0$ and $p \geq q$ we have $\Upsilon_{p}^{k}(x, y) \leq \Upsilon_{q}^{k}(x, y)$ for all $x, y \in \mathbb{R}^{n}$. Letting $k \rightarrow \infty$ in this latter inequality we deduce the desired result.

A Hölder type inequality satisfied by the map $\Upsilon_{p}(.,$.$) is recited in the$ following.

Proposition 4.5 Let $1 \leq p \leq \infty$ and $x, y, z \in \mathbb{R}^{n}$ then one has

$$
|(x, y \mid z)| \leq \Upsilon_{p}(x, z) \Upsilon_{p^{*}}(y, z) .
$$

Proof. If $y$ and $z$ are linearly dependent then the first side of (4.6) is equal to 0 and then the desired inequality is obvious. Assume that below $y$ and $z$ are linearly independent. If $p=1$ or $p=\infty$ then the inequality is obviously satisfied. Assume that $1<p<\infty$. Algorithm (4.1), with the definition of the dual, gives

$$
\Upsilon_{p}^{k+1}(x, z)=\frac{1}{p} \Upsilon_{p}^{k}(x, z)+\frac{1}{p^{*}}\left(\Upsilon_{p^{*}}^{k}\right)^{*}(x, z) \geq \frac{1}{p} \Upsilon_{p}^{k}(x, z)+\frac{1}{p^{*}} \frac{|(x, y \mid z)|}{\Upsilon_{p^{*}}^{k}(y, z)}
$$


By Young's inequality we deduce that

$$
\Upsilon_{p}^{k+1}(x, z) \geq\left(\Upsilon_{p}^{k}(x, z)\right)^{1 / p} \frac{|(x, y \mid z)|^{1 / p^{*}}}{\left(\Upsilon_{p^{*}}^{k}(y, z)\right)^{1 / p^{*}}}
$$

This, when $k \rightarrow \infty$ and after a simple reduction, yields the desired result. The proof is complete.

Following the above study, $\Upsilon_{p}(.,$.$) satisfies the condition \left(2 n_{1}\right)$. It is easy to see that the conditions $\left(2 n_{3}\right)$ and $\left(2 n_{4}\right)$ are also satisfied for $\Upsilon_{p}(.,$.$) , but$ $\left(2 n_{2}\right)$ is not obvious. We are in position to put the following open problem.

Problem 1. Prove or disprove that, for all $p \in] 1, \infty\left[, \Upsilon_{p}(.,\right.$.$) is a 2$-norm of $\mathbb{R}^{n}$.

Further, as we have seen, the map $\Upsilon_{p}(.,$.$) enjoys similar properties as that$ of $\|., .\|_{p}$. Itemizing, the following assertions have been obtained:

(i) $p \longmapsto\|., .\|_{p}$ and $p \longmapsto \Upsilon_{p}(.,$.$) are both point-wise monotone decreasing,$

(ii) $\|x, y\|_{1}=\Upsilon_{1}(x, y),\|x, y\|_{2}=\Upsilon_{2}(x, y)$ and $\|x, y\|_{\infty}=\Upsilon_{\infty}(x, y)$, for all $x, y \in \mathbb{R}^{n}$.

(iii) $\|., .\|_{p}^{*}=\|., .\|_{p^{*}}$ and $\Upsilon_{p}^{*}(.,)=.\Upsilon_{p^{*}}(.,$.$) for all 1 \leq p \leq \infty$,

(iv) $\|x, y\|_{p} \leq \frac{1}{p}\|x, y\|_{1}+\frac{1}{p^{*}}\|x, y\|_{\infty}$ and $\Upsilon_{p}(x, y) \leq \frac{1}{p}\|x, y\|_{1}+\frac{1}{p^{*}}\|x, y\|_{\infty}$, for $1 \leq p \leq \infty$ and all $x, y \in \mathbb{R}^{n}$,

(v) $|(x, y \mid z)| \leq\|x, z\|_{p}\|y, z\|_{p^{*}}$ and $|(x, y \mid z)| \leq \Upsilon_{p}(x, z) \Upsilon_{p^{*}}(y, z)$, for all $1 \leq$ $p \leq \infty$ and all $x, y, z \in \mathbb{R}^{n}$.

After this, it is natural to arise the following.

Problem 2. Prove or disprove that $\|., .\|_{p}$ and $\Upsilon_{p}(.,$.$) are algebraically$ different.

\section{References}

[1] S.S. Dragomir, Y.J. Cho, S.S. Kim and A. Sofo, Some Boas-Bellman type inequalities in 2-inner product spaces, J. Inequl. in Pure and Appl. Math. 6 (2) 55 (2005), 1-13.

[2] Y.J. Cho, P.C.S. Lin, S.S. Kim and A. Misiak, Theory of 2-Inner Product Spaces, New York, Nova Science Publishes, Inc. 2001. 
[3] S. Gähler, Linear 2-normierte Räume, Math. Nachr. 28 (1964), 1-43.

[4] M. Raïssouli and I.H.Jebril, Various Proofs of the Decrease Monotonicity of the Schatten's Power Norm, Various Families of $\mathbb{R}^{n}$-Norms and some Open Problems, International Journal of Open Problems in Computer Science and Mathematics, Vol.3, No.2 (2010), 164-174. 